\title{
Novel Approach of Edges Detection for Digital Images Based On Hybrid Types of Entropy
}

\author{
Mohamed A. El-Sayed ${ }^{1, *}$, Sayed F. Bahgat ${ }^{2}$ and S. Abdel-Khalek ${ }^{3}$ \\ ${ }^{1}$ CS Dept, CIT College, Taif University, KSA; and Math department, Faculty of Science Fayoum University, Egypt \\ ${ }^{2}$ CE Dept, CIT College, Taif University, KSA ; and Faculty of CIS, Ain Shams University, Egypt \\ ${ }^{3}$ Math Dept, Faculty of Science,Taif University, KSA; and Math Dept, Faculty of Science, Azhar University, Egypt
}

Received: 12 Jan. 2013, Revised: 15 May. 2013, Accepted: 16 May. 2013

Published online: 1 Sep. 2013

\begin{abstract}
Edges detection of digital images is used in a various fields of applications ranging from real-time video surveillance and traffic management to medical imaging applications. Most of the classical methods for edge detection are based on the first and second order derivatives of gray levels of the pixels of the original image. These processes give rise to the exponential increment of computational time. This paper shows the new algorithm based on both the Tsallis entropy and the Shannon entropy together for edge detection using split and merge technique. The objective is to find the best edge representation and minimize the computation time. A set of experiments in the domain of edge detection are presented. An edge detection performance compared to the previous classic methods, such as Canny, LOG, and Sobel. Analysis show that the effect of the proposed method is better than those methods in execution time and also is considered as easy implementation
\end{abstract}

Keywords: Edge detection, Entropy, Thresholding, Information content, Measures of information

\section{Introduction}

Edge detection has received much attention during the past two decade because of its significant importance in many research areas [1]. Since, the edge is a prominent feature of an image; it is the front-end processing stage in object recognition and image understanding system. The detection results benefit applications such as optical character recognition [2], infrared gait recognition [3,4], automatic target recognition [5], detection of video changes [6], and medical image applications [7].

Edge detection concerns localization of abrupt changes in the gray level of an image [8]. Edge detection can be defined as the boundary between two regions separated by two relatively distinct gray level properties [9]. The causes of the region dissimilarity may be due to some factors such as the geometry of the scene, the radio metric characteristics of the surface, the illumination and so on [10]. An effective edge detector reduces a large amount of data but still keeps most of the important feature of the image. Edge detection refers to the process of locating sharp discontinuities in an image. These discontinuities originate from different scene features such as discontinuities in depth, discontinuities in surface orientation, and changes in material properties and variations in scene illumination [11-13].

Many operators have been introduced in the literature, for example Roberts, Sobel and Prewitt [14-18]. Edges are mostly detected using either the first derivatives, called gradient, or the second derivatives, called Laplacien. Laplacien is more sensitive to noise since it uses more information because of the nature of the second derivatives.

Most of the classical methods for edge detection based on the derivative of the pixels of the original image are Gradient operators, Laplacien and Laplacien of Gaussian (LOG) operators [10].

Gradient based edge detection methods, such as Roberts, Sobel and Prewitts, have used two linear filters to process vertical edges and horizontal edges separately to approximate first-order derivative of pixel values of the image. Marr and Hildreth achieved this by using the Laplacien of a Gaussian (LOG) function as a filter [19]. The paper [20] classified and comparative studies of edge detection algorithms are presented. Experimental results prove that Boie-Cox, Shen- Castan and Canny operators

\footnotetext{
*Corresponding author e-mail: mas06@ fayoum.edu.eg
} 
are better than Laplacien of Gaussian (LOG), while LOG is better than Prewitt and Sobel in case of noisy image.

The paper [11] used 2-D gamma distribution, the experiment showed that the proposed method obtained very good results but with a big time complexity due to the big number of constructed masks.

To solve these problems, the study proposed a novel approach based on information theory, which is entropybased thresholding. The proposed method is decrease the computation time. The results were very good compared with the well-known Sobel gradient [21] and Canny [22] gradient results.

The outline of the paper is as follows. Image thresholding based on Shannon and Tsallis entropies is presented in section 2. Section 3, describes the proposed algorithm of edge detection. In section 4,we have presented the effectiveness of proposed algorithm in the case of real-world and synthetic images, is also, we compare the results of the algorithm against several leading edge detection methods such as Canny, LOG, and Sobel methods in the same section. Conclusion and feature work are presented in Section 5.

\section{Image thresholding based on Shannon and Tsallis entropies}

Entropy is a concept in information theory. It is used to measure the amount of information [23]. It is defined in terms of the probabilistic behavior of a source of information. In accordance with this definition, a random event $E$ that occurs with probability $P(E)$ is said to ctain [24].

$$
I(E)=\log (1 / P(E))=-\log (P(E))
$$

The amount $I(E)$ is called information content of $E$. The amount of self information of the event is inversely related to its probability. If $P(E)=1$, then $I(E)=0$ and no information is attributed to it. In this case, uncertainty associated with the event is zero. Thus, if the event always occurs, then no information would be transferred by communicating that the event has occurred. If $P(E)=0.8$, then some information would be transferred by communicating that the event has occurred. The base of the logarithm determines the unit which is used to measure the information. If the base of the logarithm is 2 , then unit of information is bit. If $P(E)=n$, then $I(E)=-\log 2\left(\frac{1}{2}\right)=1$ bit. That is, 1 bit is the amount of information conveyed when one of two possible equally likely events occurs. An example of such a situation is flipping a coin and communicating the result (Head or Tail) [25].

The basic concept of entropy in information theory has to do with how much randomness is in a signal or in a random event. An alternative way to look at this is to talk about how much information is carried by the signal.
Entropy is a measure of randomness. Consider a probabilistic experiment in which the output of a discrete source is observed during every unit of time (signaling interval). The source output is modeled as a discrete random variable $Z$. $Z$ is referred as a set of source symbols [26]. The set $Z$ of source symbols is referred to as the source alphabet, $Z=\left\{z_{1}, z_{2}, z_{3}, \ldots, z_{k}\right\}$.

The source symbol probabilities is $P=\left\{p_{1}, p_{2}, p_{3}, \ldots, p_{k}\right\}$.This set of probabilities must satisfy the condition $\sum_{i=1}^{k} p_{i}=1,0 \leq p_{i} \leq 1$. The average information per source output, denoted $S(Z)$ [27]. Shannon entropy may be described as:

$$
S(Z)=-\sum_{i=1}^{k} p_{i} \log \left(p_{i}\right)
$$

$k$ is the total number of symbols. If we consider that a system can be decomposed in two statistical independent subsystems $A$ and $B$, the Shannon entropy has the extensive property (additivity) $S(A+B)=S(A)+S(B)$, this formalism has been shown to be restricted to the Boltzmann-Gibbs-Shannon (BGS) statistics.

However, for non-extensive systems, some kind of extension appears to become necessary. Tsallis [28] has proposed a generalization of the BGS statistics which is useful for describing the thermo statistical properties of non-extensive systems. It is based on a generalized entropic form,

$$
S_{q}=\frac{1}{q-1}\left(1-\sum_{i=1}^{k} p_{i}^{q}\right)
$$

where the real number $q$ is a entropic index that characterizes the degree of non-extensivity. This expression recovers to BGS entropy in the limit $q \rightarrow 1$. Tsallis entropy has a non-extensive property for statistical independent systems, defined by the following rule [29]:

$$
S_{q}(A+B)=S_{q}(A)+S_{q}(B)+(1-q) \cdot S_{q}(A) \cdot S_{q}(B)
$$

Similarities between Boltzmann-Gibbs and Shannon entropy forms give a basis for possibility of generalization of the Shannon's entropy to the Information Theory. This generalization can be extended to image processing areas, specifically for the image segmentation, applying Tsallis entropy to threshold images, which have non-additive information content [30].

Considering $S_{q} \geq 0$ in the pseudo-additive formalism of Eq. (4), three different entropies can be defined with regard to different values of $q$. For $q<1$, the Tsallis entropy becomes a "sub extensive entropy" where $S_{q}(A+B)<S_{q}(A)+S_{q}(B)$; for $q=1$, the Tsallis entropy reduces to an standard "extensive entropy" where $S_{q}(A+B)=S_{q}(A)+S_{q}(B)$; for $q>1$, the Tsallis entropy becomes a "super extensive entropy" where $S_{q}(A+B)>S_{q}(A)+S_{q}(B)[31]$. 
Let $f(x, y)$ be the gray value of the pixel located at the point $(x, y)$. In a digital image $\{f(x, y) \mid x \in\{\$ 1,2, \ldots, N\}\}$ of size $M \times N$, let the histogram be $h(a)$ for $a=\{0,1,2, \ldots, 255\}$ with $f$ as the amplitude (brightness) of the image at the real coordinate position $(x, y)$. For the sake of convenience, we denote the set of all gray levels $\{0,1,2, \ldots, 255\}$ as $G$. Global threshold selection methods usually use the gray level histogram of the image. The optimal threshold $t^{*}$ is determined by optimizing a suitable criterion function obtained from the gray level distribution of the image and some other features of the image.

Let $t$ be a threshold value and $B=\left\{b_{0}, b_{1}\right\}$ be a pair of binary gray levels with $\left\{b_{0}, b_{1}\right\} \in G$. Typically $b_{0}$ and $b_{1}$ are taken to be 0 and 1 , respectively. The result of thresholding an image function $f(x, y)$ at gray level $t$ is a binary function $f_{t}(x, y)$ such that $f_{t}(x, y)=b_{0}$ if $f_{t}(x, y) \leq t$ otherwise $f_{t}(x, y)=b_{1}$. In general, a thresholding method determines the value $t^{*}$ of $t$ based on a certain criterion function. If $t^{*}$ is determined solely from the gray level of each pixel, the thresholding method is point dependent [27].

Let $p_{i}=p_{1}, p_{2}, p_{3}, \ldots, p_{k}$. be the probability distribution for an image with $k$ gray-levels. From this distribution, we derive two probability distributions, one for the object (class $A$ ) and the other for the background (class $B$ ), given by:

$$
p_{A}: \frac{p_{1}}{p_{A}}, \frac{p_{2}}{p_{A}}, \ldots, \frac{p_{t}}{p_{A}} \quad, \quad p_{B}: \frac{p_{t+1}}{p_{B}}, \frac{p_{t+2}}{p_{B}}, \ldots, \frac{p_{k}}{p_{B}}
$$

and where

$$
p_{A}=\sum_{i=1}^{t} p_{i} \quad, \quad p_{B}=\sum_{i=t+1}^{k} p_{i}
$$

The Tsallis entropy of order q for each distribution is defined as:

$$
\left.\begin{array}{l}
S_{q}^{A}(t)=\frac{1}{q-1}\left(1-\sum_{i=1}^{t} p_{A}^{q}\right) \\
S_{q}^{B}(t)=\frac{1}{q-1}\left(1-\sum_{i=t+1}^{k} p_{B}^{q}\right)
\end{array}\right\}
$$

The Tsallis entropy $S_{q}(t)$ is parametrically dependent upon the threshold value $t$ for the foreground and background. It is formulated as the sum each entropy, allowing the pseudo -additive property, defined in equation (3). We try to maximize the information measure between the two classes (object and background). When $S_{q}(t)$ is maximized, the luminance level $t$ that maximizes the function is considered to be the optimum threshold value [22].

$t^{*}(q)=\arg _{t \in G} \max \left[S_{q}^{A}(t)+S_{q}^{B}(t)+(1-q) \cdot S_{q}^{A}(t) \cdot S_{q}^{B}(t)\right]$.
In the proposed scheme, first create a binary image by choosing a suitable threshold value using Tsallis entropy. The technique consists of treating each pixel of the original image and creating a new image, such that $f_{t}(x, y)=0$ if $f_{t}(x, y) \leq t^{*}(q)$ otherwise $f_{t}(x, y)=1$ for every $x \in\{1,2, \ldots, M\}, y \in\{1,2, \ldots, N\}$.

When $q \rightarrow 1$, the threshold value in Equation (3), equals to the same value found by Shannon's method. Thus this proposed method includes Shannon's method as a special case. The following expression can be used as a criterion function to obtain the optimal threshold at $q \rightarrow 1$.

$$
t^{*}(1)=\arg _{t \in G} \max \left[S^{A}(t)+S^{B}(t)\right]
$$

The Eq. (8) is produce a good optimal threshold values with images of historical documents, when $q$ lies between 0 and 1 [31]. Also, this equation can be extended to fuzzy Tsallis entropy which produce a good segmentation results when nonextensive coefficient $q$ takes values between 0 to 1 . [33]

In paper [33], they were presented a thresholding technique based on two-dimensional Tsallis-Havrda-Charvat entropy with $0<q<1$. However, in most cases, when $q$ was greater than 1 , it did not produce good edge images and the threshold values produced were unacceptable.

Hence, we can chose value of $q$ lies between 0 and 1 . Take $q=0.5$ for simplifying of Eq. (8), as the following:

$$
\begin{aligned}
t^{*}(q)=\arg _{t \in G} \max \left\{S_{q}^{A}(t)+S_{q}^{B}(t)+\right. \\
\left.(1-q) \cdot S_{q}^{A}(t) \cdot S_{q}^{B}(t)\right\} . \\
t^{*}(0.5)=\arg _{t \in G} \max \left\{S_{0.5}^{A}(t)+S_{0.5}^{B}(t)+\right. \\
\left.(1-0.5) \cdot S_{0.5}^{A}(t) \cdot S_{0.5}^{B}(t)\right\} .
\end{aligned}
$$

But, from Tsallis entropy, Eq.(7) at $q=0.5$ become $S_{0.5}^{A}(t)=2 \sum_{i=1}^{t} \sqrt{p_{A}}-2, \quad$ and $S_{0.5}^{B}(t)=2 \sum_{i=t+1}^{k} \sqrt{p_{B}}-2$, then

$$
\begin{aligned}
t^{*}(0.5)= & \arg _{t \in G} \max \left\{2 \sum_{i=1}^{t} \sqrt{p_{A}}+2 \sum_{i=t+1}^{k} \sqrt{p_{B}}-4\right. \\
& \left.+\frac{1}{2}\left(2 \sum_{i=1}^{t} \sqrt{p_{A}}-2\right) \cdot\left(2 \sum_{i=t+1}^{k} \sqrt{p_{B}}-2\right)\right\} \\
t^{*}(0.5)= & \arg _{t \in G} \max \left\{2 \sum_{i=1}^{t} \sqrt{p_{A}}+2 \sum_{i=t+1}^{k} \sqrt{p_{B}}-4\right. \\
& \left.+\left(\sum_{i=1}^{t} \sqrt{p_{A}}-1\right) \cdot\left(2 \sum_{i=t+1}^{k} \sqrt{p_{B}}-2\right)\right\} .
\end{aligned}
$$




$$
\begin{aligned}
t^{*}(0.5)= & 2 \arg _{t \in G} \max \left\{\sum_{i=1}^{t} \sqrt{p_{A}}+\sum_{i=t+1}^{k} \sqrt{p_{B}}-2\right. \\
& \left.+\left(\sum_{i=1}^{t} \sqrt{p_{A}}-1\right) \cdot\left(\sum_{i=t+1}^{k} \sqrt{p_{B}}-1\right)\right\} . \\
t^{*}(0.5)= & 2 \arg _{t \in G} \max \left\{\sum_{i=1}^{t} \sqrt{p_{A}}+\sum_{i=t+1}^{k} \sqrt{p_{B}}-2\right. \\
& \left.+\sum_{i=1}^{t} \sqrt{p_{A}}\left(\sum_{i=t+1}^{k} \sqrt{p_{B}}-1\right)-\sum_{i=t+1}^{k} \sqrt{p_{B}}+1\right\} . \\
t^{*}(0.5)= & 2 \arg _{t \in G} \max \left\{\sum_{i=1}^{t} \sqrt{p_{A}} \sum_{i=t+1}^{k} \sqrt{p_{B}}-1\right\} .
\end{aligned}
$$

By removing the constant values, $t^{*}(q=0.5)$ is tend to the following equation:

$$
t^{*}(q=0.5)=\arg _{t \in G} \max \left\{\sum_{i=0}^{t} \sqrt{\frac{p_{i}}{p_{A}}}+\sum_{i=t+1}^{255} \sqrt{\frac{p_{i}}{p_{B}}}\right\} .
$$

The Threshold procedure to select suitable threshold value $t^{*}$ and $q=0.5$ for grayscale image $f$ can now be described as follows:

\section{Procedure Threshold,}

Input: $A$ grayscale image $f$ of size $r \times c$.

Output: $t^{*}$ of $f$, for $q=0.5$.

Begin

1. Let $f(x, y)$ be the original gray value of the pixel at the point $(x, y), x=1 . . r, y=1 . . c$.

2. Calculate the probability distribution $0 \leq p_{i} \leq 255$.

3. For all $t \in\{0,1, \ldots, 255\}$,

i. Calculate $p_{A}, p_{B}, p_{A}$ and $p_{B}$ using Eq.s (5 and 6). End.

ii. Find optimum threshold value $t^{*}$, using Eq. (10).

The technique consists of treating each pixel of the original image and creating a new image, such that $f_{t}(x, y)=0$ if $f_{t}(x, y) \leq t^{*}(q)$ otherwise $f_{t}(x, y)=1$ for every $x \in\{1,2, \ldots, M\}, y \in\{1,2, \ldots, N\}$.

\section{The proposed algorithm}

This section presents the concept of object connectivity. It introduces a technique of edge detection based on entropy and geometric properties of the object. Geometric properties such as connectivity, projection, area, and perimeter are important components in binary image processing. An object in a binary image is a connected set of pixels. In what follows, we present some definitions related to connectivity of pixels in a binary image.
- $\quad$ Connected Pixels:A pixel $f_{0}$ at $\left(i_{0}, j_{0}\right)$ is connected to another pixel $f_{n}$ at $\left(i_{n}, j_{n}\right)$ if and only if there exists a path from $f_{0}$ to $f_{n}$ which is a sequence of points $\left(i_{0}, j_{0}\right),\left(i_{1}, j_{1}\right), \ldots,\left(i_{n}, j_{n}\right)$, such that the pixel at $\left(i_{k}, j_{k}\right)$ is a neighbor of the pixel at $\left(i_{k+1}, j_{k+1}\right)$ and $f_{k}=f_{k}+1$ for all, $0<k<n-1$.

- 4-connected: When a pixel at location $(i, j)$ has four immediate neighbors at $(i+1, j),(i-1, j),(i, j+l)$, and $(i, j-1)$, or four immediate neighbors at $(i+1, j+1),(i-1, j+1),(i+1, j-1)$, and $(i-1, j-1)$ they are known as,4-connected. Two four connected pixels share a common boundary as shown in Figure (1-a,1-b).

- 8-connected: When the pixel a $t \operatorname{location}(i, j)$ has. in addition to above two types of four immediate neighbors, together, they are known as 8-connected. Thus two pixels are eight neighbors if they share a common corner. This is shown in Figure (1-c).

- $\quad$ Connected component: A set of connected pixels (4 or 8 connected) forms a connected component. Such a connected component represents an object in a scene as shown in Figure (1-d).

In order to obtain edge detection, we find classification of all pixels that satisfy the criterion of homogeneousness, and detection of all pixels on the borders between different homogeneous areas. In the proposed scheme, first create a binary image by choosing a suitable threshold value using Tsallis entropy, using of the Threshold procedure. Region labeling in this system is done using 4-neighbor or 8-neighbor connectivity. A common alternative would be to use 4-neighbor connectivity instead (Figure 1).

The Edge Detection Procedure can be described as follows (using the 4-connected or diagonal 4-connected):

Procedure Edge Detection;

Input: A grayscale image Aof size $r \times c$ and $t^{*}$.

Output: The edge detection image $g$ of $A$.

Begin

Step 1: Create a binary image: For all $x, y$,

If $f(x, y) \leq t^{*}$ then $f(x, y)=0$ Else $f(x, y)=1$.

Step 2: Initialization of the output edge image of size $r \times c, g(x, y)=0$ and for all $x$ and $y$.

Step 3: Checking for edge pixels:

For all $1<j<r$, and $1<i<c$ do

$$
\begin{aligned}
& \delta_{1}=\left|f_{j, i}-f_{j, i-1}\right|+\left|f_{j, i}-f_{j, i+1}\right|, \\
& \delta_{2}=\left|f_{j, i}-f_{j-1, i}\right|+\left|f_{j, i}-f_{j+1, i}\right|, \\
& \varepsilon_{1}=\left|f_{j, i}-f_{j-1, i-1}\right|+\left|f_{j, i}-f_{j+1, i+1}\right|, \\
& \varepsilon_{2}=\left|f_{j, i}-f_{j-1, i+1}\right|+\left|f_{j, i}-f_{j+1, i-1}\right|, \\
& \text { If } \delta_{1}+\delta_{2}=0 \text { or } \varepsilon_{1}+\varepsilon_{2}=0 \text { then } g_{i j}=1 .
\end{aligned}
$$$$
\text { End }
$$

End Procedure.

The steps of proposed algorithm are as follows: 


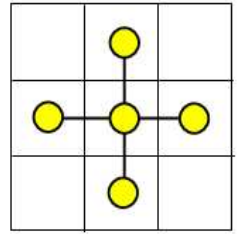

(a)

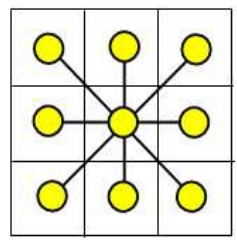

(c)

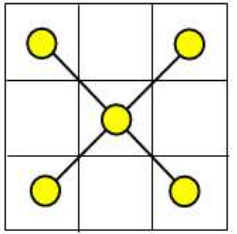

(b)

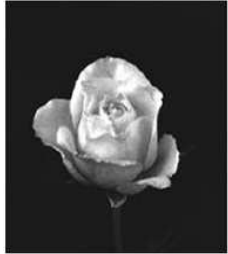

(a) Original image

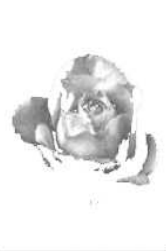

(b) Part1(object)

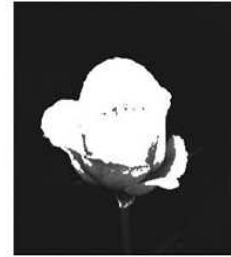

(c) Part2(background)
000000000000000000 000000001111100000 000011111111000000 000011111110000000 000111111110000000 000000111100000000

(d) 000000000000000000

Fig. 1: (a) 4-connected, (b) Diagonal 4-connec3ted, (c) 8connected, and (d) Connected component.

Step 1: We use Shannon entropy, the equation (2), to find the global threshold value $\left(t_{1}\right)$. The image is split by $t_{1}$ into two parts, the object and background. See Figure 2.

Step 2: We use Tsallis entropy, the equation (10), $q=0.5$. Applying the equation (10), to find the locals threshold values $t_{2}$ and $t_{3}$ of object and background, respectively.

Step 3: Applying EdgeDetection Procedure with thresh-old values $t_{1}, t_{2}$ and $t_{3}$. See Figure 3 a-c.

Step 4: Merge the resultant images of Step 3 to obtain the reconstructed edge image. See Figure 3.d

We note that, the proposed algorithm split the original image into two grayscale parts in Step 1. The threshold values $\left(t_{2}\right)$ and $\left(t_{3}\right)$ are global threshold of the object and background parts, respectively. But they are locals relative to the original gray scale image. The proposed algorithm merge the output of binary sub-edge images into edge image related to original image.

In order to minimize the execution time, we deal with the histogram vectors, $0,1, \ldots, t_{1}$ and $t_{1}+1, \ldots, 255$ of object and background parts, respectively rather than the matrices size of them.

\section{Results and Discussion}

In order to test the method proposed in this paper and compare with the other edge detectors, common gray level test images with different resolutions and sizes are detected by Canny, LOG, and Sobel and the proposed

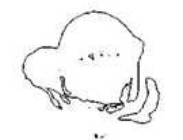

(a) At $t_{1}$ of original image

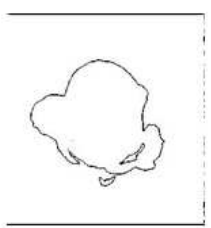

(c) At $t_{3}$ of background part (d) reconstructed edge image.
Fig. 2: Splitting the original image into two parts, object and a background.

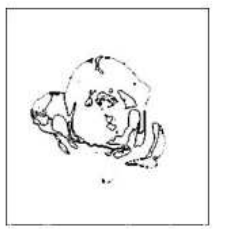

(b) At $t_{2}$ of object part

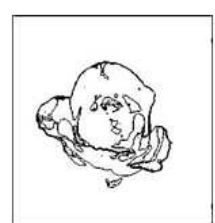

Fig. 3: Edge images of original image, object and background parts, then reconstructed edge image

method respectively. The performance of the proposed scheme is evaluated through the simulation results using MATLAB. Prior to the application of this algorithm, no pre-processing was done on the tested images.

The algorithm has two main phases - global and local enhancement phase of the threshold values and detection phase, we present the results of implementation on these images separately. Here, we have used in addition to the original gray level function $f(x, y)$, a function $g(x, y)$ that is the average gray level value in a $3 \times 3$ neighborhood around the pixel $(x, y)$.

The proposed algorithm used the good characters of each Shannon entropy and Tsallis entropy, together, to calculate the global and local threshold values. Hence, we 


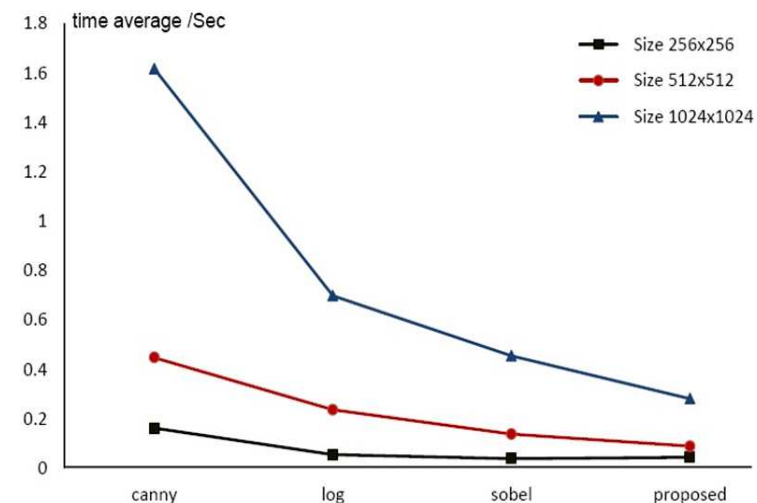

Fig. 4: Run time of Canny, LOG, Sobel, and proposed method with different size test images

ensure that the proposed algorithm done better than the algorithms that based on Shannon entropy or Tsallis entropy separately.

We using MATLAB to calculate the average time for each method at different images size by repeating 10 times for each type of image. As shown in Figure 4, The chart of the test images and the average of run time for the classical methods and proposed scheme. It has been observed that the proposed edge detector works effectively for different gray scale digital images as compare to the run time of classical methods.

Some selected results of edge detections for these test images using the classical methods and proposed scheme are shown in Figures 5-8.

Analysis method of the results is done such as found in $[25,33,34]$. It is based on how much information is lost due to thresholding. In this analysis, given two edge images based on thresholding of a same original image, we prefer the one which lost the least amount of information. From the results; it has again been observed that the proposed method works well as compare to the previous methods, LOG and Sobel (with default parameters in MATLAB).

Majority of computational operations of proposed method are run on the histogram with limited size ( at most 256 values), that is decrease the execution time of the proposed method. The operations of previous methods runs on all pixels of image with $r \times c$. However with large size of images, the previous methods take a long time about the proposed method (See Procedure Threshold in section 2 and Figure 4).

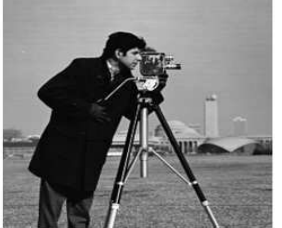

Original image

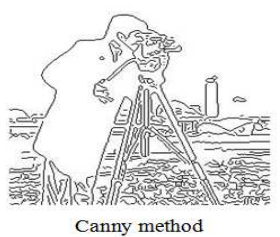

Canny method

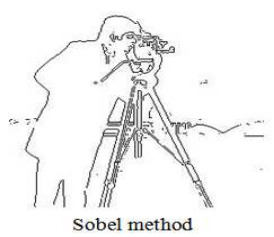

Sobel method

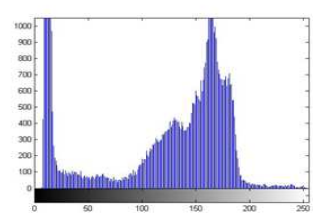

Histogram

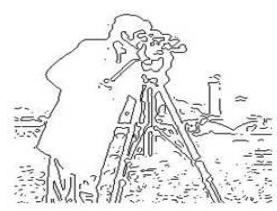
LOG method

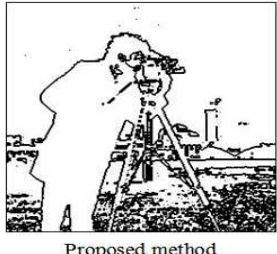

Proposed method
Fig. 5: Cameraman image with $256 \times 256$ pixel

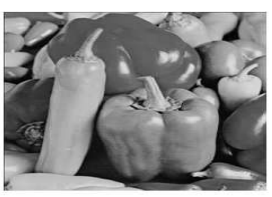

Original image

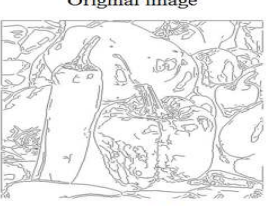

Canny method

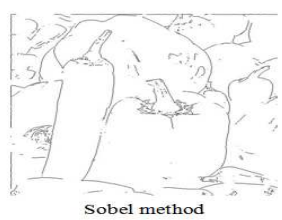

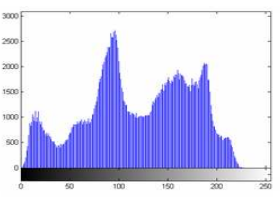

histogram

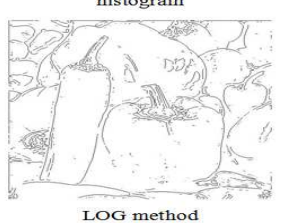

LOG method

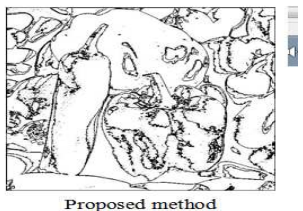

Fig. 6: Peppers image with $512 \times 512$ pixel 

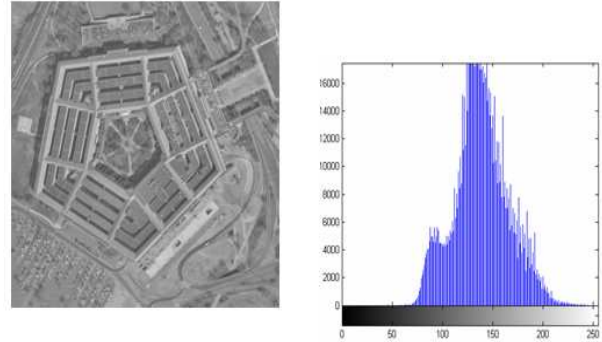

Original image

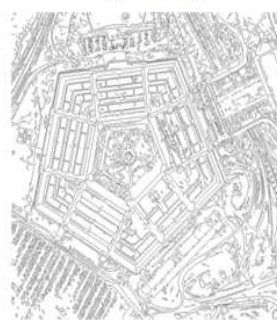

Canny method

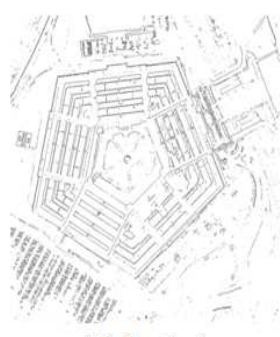

Sobel method

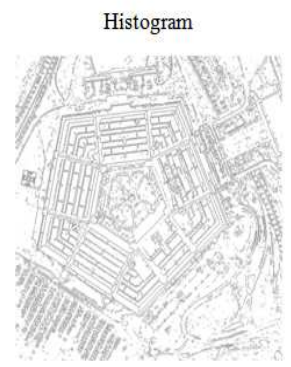

LOG method

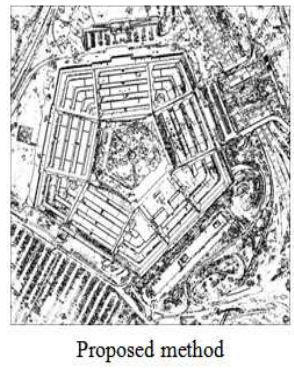

Fig. 7: pentagon image with $1024 \times 1024$ pixel

\section{Conclusion and Feature Work}

This paper shows the new algorithm based on both the Tsallis entropy and the Shannon entropy together for edge detection using split and merge technique. The objective is to find the best edge representation and minimize the computation time. A set of experiments in the domain of edge detection are presented. An edge detection performance is compared to the previous classic methods, such as Canny, LOG, and Sobel. Analysis show that the effect of the proposed method is better than those methods in execution time, also is considered as easy implementation. The significance of this study lies in decreasing the computation time with generate suitable quality of edge detection. In this way, the hybrid entropic edge detector presented in this paper uses both Shannon entropy and Tsallis entropy. It is already pointed out in

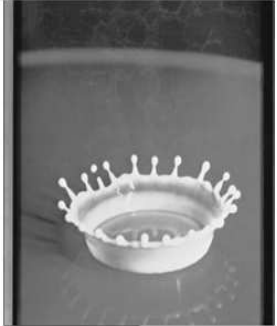

Original image

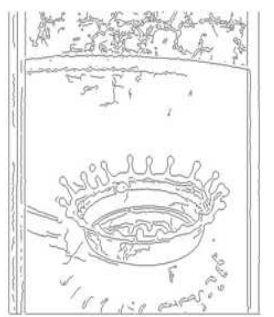

Canny method

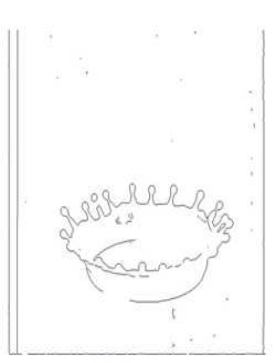

Sobel method

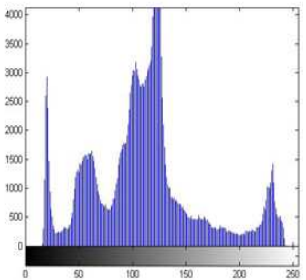

Histogram

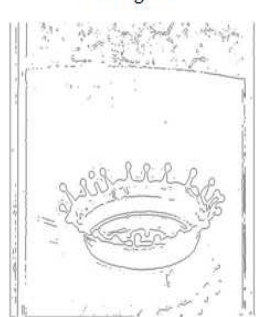

LOG method

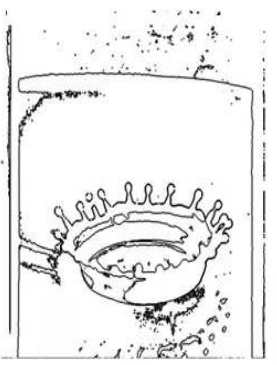

Proposed method
Fig. 8: image with $512 \times 512$ pixel

the introduction that the traditional methods give rise to the exponential increment of computational time.

Experiment results have demonstrated that the proposed scheme for edge detection can be used for different gray level digital images. Another benefit comes from easy implementation of this method. An important future investigation will be the study of edge detection in the case of medical image.

\section{Acknowledgement}

The authors gratefully acknowledge the Taif University for the financial support (Project No: 1-433-1835). Also, the authors would like to thank the reviewers for their comments, which have greatly assisted them in improving the quality of the presented work. 


\section{References}

[1] Yuan-Hui Yu and Chin-Chen Chang,. A new edge detection approach based on Image Context Analysis. Int. J. Image Vision Comput, 24, 1090-1102 (2006).

[2] J. Lzaro, J. L. Martn, J. Arias, A. Astarloa, C. Cuadrado, Neuro semantic thresholding using OCR software for high precision OCR applications. Image Vision Comput., 28, 571-578, (2010).

[3] Z. Xue, D. Ming, W. Song, B. Wan, S. Jin, Infrared gait recognition based on wavelet transform and support vector machine. Patt. Recog, 43, 2904-2910 (2010).

[4] Y. Zhang, L. Wu, Optimal Multi-Level Thresholding Based on Maximum Tsallis Entropy via an Artificial Bee Colony Approach, Entropy, 13, 841-859 (2011).

[5] G. C. Anagnostopoulos, SVM-based target recognition from synthetic aperture radar images using target region outline descriptors. Nonlinear Anal.-Theor. Meth. App., 71, e2934e2939 (2009).

[6] Y. T. Hsiao, C. L. Chuang, Y. L. Lu, J. A. Jiang, Robust multiple objects tracking using image segmentation and trajectory estimation scheme in video frames. Image Vision Comput., 24, 1123-1136 (2006).

[7] M. T. Doelken, H. Stefan, E. Pauli, A. Stadlbauer, T. Struffert, T. Engelhorn, G. Richter, O. Ganslandt, A. Doerfler, T. Hammen, 1H-MRS profile in MRI positiveversus MRI negative patients with temporal lobe epilepsy. Seizure, 17, 490-497 (2008).

[8] S. Kresic-Juric, , D. Madej and S. Fadil. Applications of Hidden Markov Models in Bar Code Decoding. Intl. J. Patt. Recog. letters, 27, 1665-1672 (2006).

[9] G. Markus, Essam A. EI-Kwae and R. K. Mansur. Edge detection in medical images using a genetic algorithm. IEEE Trans. on Medical Imaging, 17, 469-474 (1998).

[10] M. Wang and Y. Shuyuan, "A Hybrid Genetic Algorithm Based Edge Detection Method for SAR Image", In: IEEE Proceedings of the Radar Conference'05, 1503-506 (2005).

[11] A. El-Zaart, "A Novel Method for Edge Detection Using 2 Dimensional Gamma Distribution", J. of Comput. Sc., 6, 199-204 (2010).

[12] M. A. El-Sayed , "A New Algorithm Based Entropic Threshold for Edge Detection in Images", Int. J. of Comp. Sc., 8, 71-78, (2011).

[13] M. A. El-Sayed, Edges Detection Based On Renyi Entropy With Split/Merge. Comp. Eng. And Intel. Sys. (CEIS), 3, 32-41 (2012).

[14] V. Aurich, and J. Weule, "Nonlinear Gaussian filters performing edge preserving diffusion. ", Proceeding of the 17th Deutsche Arbeitsgemeinschaft für Mustererkennung (DAGM) Symposium, Sept. 13-15, Bielefeld, Germany, Springer-Verlag, 538-545 (1995).

[15] M. Basu, "A Gaussian derivative model for edge enhancement.", Patt. Recog., 27, 1451-1461 (1994).

[16] G. Deng, and L. W. Cahill, "An adaptive Gaussian filter for noise reduction and edge detection.", Proceeding of the IEEE Nuclear Science Symposium and Medical Imaging Conference, Oct. 31-Nov. 6, IEEE Xplore Press, San Francisco, CA., USA, 1615-161 (1993).

[17] C. Kang, and W. Wang, "A novel edge detection method based on the maximizing objective function.", Patt. Recog., 40, 609-618 (2007).
[18] Q. Zhu, "Efficient evaluations of edge connectivity and width uniformity.", Image Vis. Comput., 14, 21-34 (1996).

[19] B. Mitra, "Gaussian Based Edge Detection Methods- A Survey ". IEEE Trans. on Systems, Manand Cybernetics , 32, 252-260 (2002).

[20] M. Roushdy, "Comparative Study of Edge Detection Algorithms Applying on the Grayscale Noisy Image Using Morphological Filter", GVIP, Special Issue on Edge Detection, 51-59 (2007).

[21] R. C. Gonzalez, and R. E. Woods, "Digital Image Processing.", 3rd Edn., Prentice Hall, New Jersey, USA. ISBN: 9780131687288, 954 (2008).

[22] J. Canny, "A computational approach to edge detection.", IEEE Trans. Patt. Anal. Mach. Intell., 8, 679-698 (1986).

[23] C. E. Shannon, A mathematical theory of communication. Int. J. Bell. Syst. Technical, 27, 379-423 (1948).

[24] M. A. El-Sayed, and T. Abd-El Hafeez, "New Edge Detection Technique based on the Shannon Entropy in Gray Level Images", Int. J. on Comput. Sc. and Eng. (IJCSE), 3, 2224-2232 (2011).

[25] B. Singh and A. P. Singh, " Edge Detection in Gray Level Images Based on the Shannon Entropy", J. Comput. Sci., 4, 186-191 (2008).

[26] S. Haykin. Communication System. John Wiley and Sons New York Publishers, 614- 621 (1995).

[27] F. Luthon, M. Lievin and F. Faux, "On the use of entropy power for threshold selection.” Int. J. Signal Proc., 84, 17891804 (2004)

[28] C. Tsallis, "Possible generalization of Boltzmann-Gibbs statistics," J. Stat. Phys., 52, 479-487 (1988).

[29] M. P. de Albuquerque, I. A. Esquef , A.R. Gesualdi Mello, "Image Thresholding Using Tsallis Entropy." Patt. Recog. Letters, 25, 1059-1065 (2004).

[30] M. A. El-Sayed , S. Abdel-Khalek , and E. Abdel-Aziz, "Study of Efficient Technique Based On 2D Tsallis Entropy For Image Thresholding", Int. J. on Comp. Sc. and Eng. (IJCSE), 3, 3125-3138 (2011).

[31] C. A. B. Mello, L. A.Schuler, Thresholding Images of Historical Documents Using a Tsallis-Entropy Based Algorithm, J. of software, 3, 29-36 (2008).

[32] Y. Tang, Q. Di, X. Guan, F. Liu, Threshold Selection Based on Fuzzy Tsallis Entropy and Particle Swarm Optimization, NeuroQuantology, 6, 412-419 (2008).

[33] P. K. Sahoo , G. Arora, Image thresholding using twodimensional Tsallis-Havrda-Charvat entropy, Patt. Recog. Letters , 27, 520-528 (2006).

[34] P. K. Sahoo, G. Arora, A thresholding method based on twodimensional Renyi's entropy, Patt. Recog., 37, 1149-1161 (2004). 


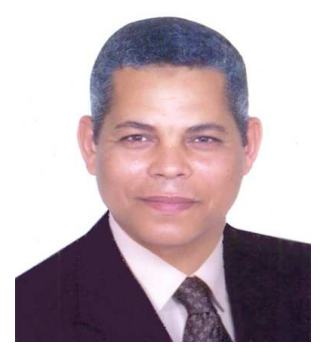

Mohamed A. El-Sayed has obtained his $\mathrm{PhD}$ degree in Computer Science in 2007 from Minia University, Egypt. His research interests include image processing, and graph drawing. $\mathrm{He}$ is the author of several articles published in reputed journals and is a member of different working groups. He is a lecturer in Math. Department, Faculty of Science, Fayoum University, Egypt. He is presently employed as Computer Science Assistant Professor at Computers and Information Technology College, Taif University, KSA.

Sayed F. Bahgathas

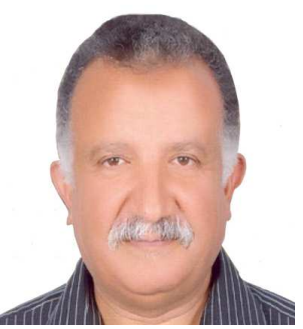
obtained his $\mathrm{PhD}$ degree in Electrical Engineering in 1989 from Illinois Institute of Technology, Chicago, Illinois, USA. His research interests include intelligent computing, signal processing, and image processing. He is the author of several articles published in reputed journals and is a member of different working groups. He is Professor, Scientific Computing Department, Faculty of Computer and Information Science, Ain Shams University, Egypt. $\mathrm{He}$ is presently employed as Professor of Computer Engineering at Computers and Information Technology College, Taif University, KSA.

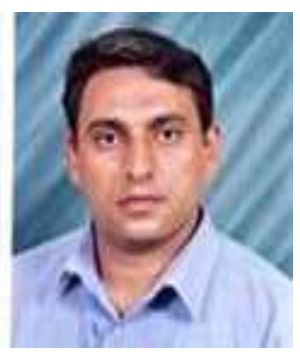

S. Abdel-Khalek has obtained his $\mathrm{PhD}$ degree in Quantum information in 2004 from Azhar University. His research interests include different directions in quantum information processing. He is the author of several articles published in different international scientific journals and is a member of different working groups. He is Assistant Professor, of Applied Mathematics, Mathematics Department, Faculty of Science, Sohag University, Egypt. $\mathrm{He}$ is presently employed as Assistant Professor of Mathematics and Statistics Department, Faculty of Science, Taif University, KSA. 